\title{
Nivel de sesgos cognitivos de representatividad y confirmación en estudiantes de Psicología de tres universidades del Bío-Bío
}

\section{Level of Cognitive Biases of Representativeness and Confirmation in Psychology Students of three Bío-Bío Universities}

\author{
Alexandra Castro $M$. \\ Universidad del Bío-Bío, Chillán, Chile \\ ORCID: https://orcid.org/0000-0002-4522-2083 \\ Zabrina Hernández A. \\ Universidad del Bío-Bío, Chillán, Chile \\ ORCID: https://orcid.org/0000-0002-7510-5611 \\ Elisa Riquelme M. \\ Universidad del Bío-Bío, Chillán, Chile \\ ORCID: https://orcid.org/0000-0002-8939-8478 \\ Carlos Ossa C.* \\ Universidad del Bío-Bío, Chillán, Chile \\ ORCID: https://orcid.org/0000-0002-2716-2558 \\ Jaime Aedo S. \\ Universidad Santo Tomás, Los Ángeles, Chile \\ ORCID: https://orcid.org/0000-0002-1739-5446

\section{Silvia Da Costa D.} \\ Universidad del País Vasco, San Sebastián, España \\ ORCID: https://orcid.org/0000-0001-6011-821X

\section{Darío Páez R.} \\ Universidad del País Vasco, San Sebastián, España \\ ORCID: https://orcid.org/0000-0002-8459-6037
}

Recibido 30-09-18 Revisado 25-10-18 Aprobado 19-01-19 En línea 12-03-19

*Correspondencia

Email: cossa@ubiobio.cl
Citar como:

Castro, A. Hernández, Z., Riquelme, E., Ossa, C., Aedo, J., Da Costa, S., Páez, D. (2019). Nivel de sesgos cognitivos de representatividad y confirmación en estudiantes de Psicología de tres universidades del BíoBío. Propósitos y Representaciones, 7(2), 210-239. doi: http://dx.doi.org/10.20511/pyr2019.v7n2.245 


\section{Resumen}

Los sesgos cognitivos son formas no razonadas de tomar decisiones, a menudo de manera parcializada. El objetivo de esta investigación fue describir el nivel de sesgos de representatividad y confirmación en estudiantes de psicología chilenos, para conocer cómo se presenta este fenómeno. Los participantes fueron 198 estudiantes de psicología, de tres universidades del sur de Chile. El instrumento se basó en dos tareas cognitivas derivadas de Kanheman y Tversky; se analizaron los datos con estadística descriptiva y diferencias de medias. Los resultados indican alto nivel de sesgos en los grupos de estudiantes, en las dos tareas evaluadas; el sesgo de representatividad presenta diferencias entre universidades, solo en la subtarea 1, así como en la tarea 2, relacionada con sesgo de confirmación; así mismo se observan diferencias en sesgo de representación según edad y sexo. Se concluye la necesidad de fortalecer habilidades de razonamiento en la formación inicial de los futuros psicólogos y psicólogas, con la finalidad de mejorar las habilidades relacionadas con la toma de decisiones en el campo profesional.

Palabras Claves: Sesgos; Cognición; Formación profesional; Psicología

\section{Summary}

Cognitive biases are unreasonable ways of making decisions, often in a one-sided way. The objective of this research was to describe the level of representativeness and confirmation biases in Chilean psychology students, in order to know how this phenomenon occurs. The participants were 198 psychology students from three universities in southern Chile. The instrument was based on two cognitive tasks derived from Kanheman and Tversky. The data were analyzed through descriptive statistics and mean differences. The results indicate a high level of biases in the groups of students, in the two tasks evaluated; the representativeness bias presents differences among universities only in sub-task 1 , as well as in task 2 , related to confirmation bias. Likewise, there are differences in representation bias according to age and sex. The need to strengthen reasoning skills in the initial training of future psychologists, in order to improve skills related to decision making in the professional field is concluded.

Keywords: Biases; Cognition; Professional Training; Psychology

\section{Introducción}

En los últimos años el término heurística, y específicamente el de sesgos cognitivos, es un tema que cada vez se hace más frecuente, sin embargo, sus estudios se enfocan en su mayoría en áreas tales como ingeniera o marketing (Rodríguez, 2012). Si bien en sus comienzos el concepto era entendido desde un punto de vista probabilístico, de a poco se ha ido acercando al área de la psicología, a través de los estudios realizados por el psicólogo y economista Daniel Kahneman y el psicólogo cognitivo y matemático Amos Tversky. Ellos han realizado variadas investigaciones donde presentan el concepto de sesgos cognitivos, definiéndolos como formas no razonadas en que se toman las decisiones, permitiendo hacer deducciones sin caer en un alto nivel de esfuerzo, lo que podría desencadenar que la toma de decisiones sea de manera tendenciosa o errada (Tversky \& Kahneman, 1983).

Esta investigación busca relacionar el tema de los sesgos con la formación de psicólogos/as, pues este profesional, en su papel de apoyo a la comunidad está sujeto a una gran influencia en las personas, por lo cual es preciso no verse influenciado por un arraigo excesivo de sesgos ante cualquier tipo de intervención, ya que esto perjudica negativamente la toma de decisiones. Se debe tener presente lo fundamental que es para este profesional, la toma de decisiones en su desempeño laboral, así como en todo ámbito de desarrollo (Juliá, 2013), ya que cada decisión que tome, debe ser consciente y libre de la influencia; el efecto que los sesgos provocan en dichas decisiones, podría desencadenar repercusiones importantes y determinantes, afectando de manera negativa la relación profesional y por ende, la eficacia a la que aspira el/la psicólogo/a al ejercer 
la profesión. Sin embargo, no se ha logrado encontrar estudios que analicen cómo estos profesionales manejan sesgos y heurísticos en su desempeño profesional, y tampoco como se trabajan estas habilidades en la formación universitaria en Chile.

Específicamente, en la presente investigación, se trabajó con el sesgo de representatividad y con el sesgo de confirmación; el primero es entendido como la probabilidad de que algo ocurra, en base a cuanto representa o se asemeja a nuestras creencias previas, ignorando otra información útil respecto a la situación o a la problemática (Kahneman, Slovic \& Tversky, 1982); el sesgo de confirmación se presenta como la tendencia general a buscar o seleccionar la información que confirme hipótesis previas, siendo resistentes a información que la contradiga (Páez, Villarreal, Echeverría \& Valencia, 1987; Nieto, 2002).

Las personas tienden a decidir rápidamente ante diferentes situaciones, atribuyendo que la situación en curso tendrá un solo significado, ignorando que se elige por una alternativa frente a otras, esto se llama tendencia a la rigidez cognitiva prematura, lo que lleva al error, pues muchas veces estas interpretaciones no son correctas; al tener una idea previa de cómo funciona el mundo o el tener una imagen de otros en la mente, no se examina información nueva o reexamina la información antigua, sino, que en vez de ello, se asimila la información de acuerdo a las ideas preexistentes, con lo que éstas se refuerzan aún más (Martín \& Alvarez, 2000; Rivas, 2008).

Tener la facultad como persona de poder discernir entre una serie de alternativas está estrechamente relacionado con el proceso de toma de decisiones, aunque solo sea una parte de éste, pues dicho proceso se lleva a cabo a través de varias fases que, según Cortada (2008) se presentan primeramente desde la identificación de la información, para luego definir el proceso de decisión y así generar una serie de alternativas y valorar estas alternativas; posteriormente se elegirá la que mejor resuelva el problema valorando los resultados del proceso y el fin de éste. Por lo tanto, si se desea tomar una buena decisión o la mejor acción frente a una interrogante, es recomendable tener en cuenta estos pasos como la base de discriminación y ordenamiento de las opciones, las que eventualmente servirán para saciar, de alguna forma, la incertidumbre que nos provoca la elección de alternativas para un fin determinado.

Se ha de tener siempre en cuenta que, a la hora de tomar una decisión frente a una problemática que genera incertidumbre, la persona se encuentra irremediablemente sujeta a la valoración de las alternativas, fase en la cual se evalúan las consecuencias que pueda traer consigo cada una de esas opciones, ya sean positivas o negativas, para luego elegir la alternativa más acertada a la solución del problema de decisión, con lo que finalmente se pretende valorar los resultados del proceso, en relación con la efectividad de la resolución del problema, siempre evaluando cada una de las fases mencionadas anteriormente (Nieto, 2002).

A través de los estudios realizados, se han precisado dos maneras de realizar la toma de decisiones, cada una de ellas orientada a diferentes definiciones, una conocida como algoritmos y la otra como heurísticos. Ante la clasificación e interpretación de problemas, el primer paso para solucionarlos es mediante la selección de pasos que más acomode, o sean más conocidos para el individuo, lo cual es llamado algoritmos (Morris \& Maisto, 2001). Al elegir la estrategia de algoritmos, se garantiza la solución ya que se presentan resultados absolutamente correctos, debido a que plantean la efectiva resolución de problemas a través de una secuencia fija de pasos con un correcto razonamiento, en donde se asegura el objetivo; éstos, se utilizan masivamente en las ciencias exactas y la matemática.

Por otra parte, no todos los problemas se pueden resolver con algoritmos, para estos casos en que no se sabe si los resultados que serán obtenidos serán del todo correcto, o no se tiene certeza de la posible solución al problema se recurre a la heurística, entendida como un conjunto de reglas simples que quizás no nos ayuden a resolver el problema, pero al menos pone las ideas a nuestro alcance, dependiendo del método heurístico que se utilice para cada interrogante, logrando con ello que se pueda agilizar o entorpecer la solución (Morris \& Maisto, 2001). 
Kahneman y Tversky (citado en Cortada, 2008) realizan una descripción de heurísticos, que se presentan como variados juicios intuitivos bajo incertidumbre, mencionando que:

Aunque la intuición heurística se distingue de los procesos de razonamiento formativo por pautas de juicios sesgados, los heurísticos en sí mismos son procedimientos de estimación que de ningún modo son irracionales. Son respuestas intuitivas normales, no solo para los problemas de alta complejidad, sino para las más simples cuestiones de verosimilitud, frecuencia y predicción (p.69).

El concepto heurísticas, según Muñoz (2011), es una herramienta de "ahorro de energía" (p.6), debido a que permite tomar una decisión sin gran esfuerzo y, por lo general, funciona de manera aceptable. Sin embargo, cuando estas heurísticas no llevan a una decisión acertada, se convierten en sesgos, lo que produce que las personas tomen decisiones basándose exclusivamente en sus preconceptos, sin utilizar un razonamiento óptimo.

Así mismo, Kahneman (citado en Páez et al., 1987) define la heurística como reglas simples y eficientes, resultado de procesos evolutivos o de aprendizaje, propuestos para explicar cómo las personas toman decisiones y resuelven problemas, por lo general complejos, en base a información incompleta, adaptando sus conocimientos a la información nueva propuesta, sin mayor esfuerzo cognitivo en el proceso. Cuando se utilizan las heurísticas para resolver problemas complejos, estas suceden muchas veces de manera automática, lo que da lugar a sesgos cognitivos. Dichos términos suelen ser igualados en la vida cotidiana, sin embargo, una definición teórica adecuada lleva a entenderlos y diferenciarlos de manera concreta, comprendiendo la heurística como un concepto global, del cual se desprenden los sesgos cognitivos (Tversky \& Kahneman, 1983); a pesar de existir una gran variedad de sesgos (Nieto, 2002), en este estudio se consideraron solo dos, el sesgo de representatividad y el de confirmación.

El sesgo de representatividad, es definido como un juicio sobre la relación existente entre una muestra y una población, que hace pensar que un evento es probable si nos parece representativo de una clase mayor (Martín \& Álvarez, 2000); por ello, puede investigarse empíricamente con las personas, conociendo cuáles son sus juicios representativos frente alguna situación y/o problemática; además, es entendido como la similitud de una descripción con los estereotipos, ignorando las tasas base y las dudas sobre la veracidad de la descripción. De este modo, se juzga la probabilidad de que algo ocurra, con base en cuanto representa, o se parece a nuestras creencias previas, ignorando otra información útil respecto a la situación o al problema. Es así como se puede llegar a ignorar elementos fundamentales en la toma de decisiones, viéndose influidos por acontecimientos previos (Tversky \& Kahneman, 1983).

El sesgo de confirmación fue acuñado por el psicólogo inglés Peter C. Watson (1960, citado en Rodríguez, 2012), a través de un experimento realizado a sujetos a los cuales desafió para que identificaran una regla que se aplica a tres números pares consecutivos (dos, cuatro y seis), señalando que cumplían con la regla. Lo que se esperaba era que los sujetos fueran capaces de generar sus propias series esperando la confirmación del investigador para que corrobora si cumplían o no con la regla. Watson comprobó que los sujetos buscaban solo ejemplos positivos, para confirmar a través de las secuencias su hipotética regla (Rodríguez, 2012). En relación con lo mencionado, el término se puede definir como la tendencia general a buscar o seleccionar la información que confirme hipótesis previas, por lo que las personas tienden tomar en cuenta la información que confirman sus hipótesis, siendo resistentes a información que las contradiga (Páez et al., 1987). Esto se puede reflejar en la tendencia a utilizar sólo la información que está más accesible, sin hacer mayor esfuerzo por incorporar nuevos esquemas o información a la situación que se pretende analizar.

Los sesgos, además, están relacionados con los prejuicios, pues éstos se caracterizan por su fácil adquisición e instauración, así como también por ser muy operativos (Martín \& Álvarez, 2000), lo que desencadena una fácil transmisión de individuo en individuo, siendo por tanto 
difíciles de modificar. Los prejuicios proporcionan una idea vaga sobre algo que muchas veces desconocemos, por eso la experiencia se presenta como un factor favorable para lograr el desprejuicio, debido a que es la encargada de otorgar información nueva respecto a los esquemas anteriormente instaurados (Olmo, 2005).

Se hace imperante reconocer que el/la psicólogo/a trabaja diariamente con personas que se encuentran, en momentos vitales de suma vulnerabilidad, en los cuales, las características sociales y culturales de pacientes pueden verse juzgadas negativamente si no se tiene en consideración el impacto de los sesgos en la toma de decisiones.

Tomando en cuenta los factores mencionados por Díaz-Lázaro (2011) tales como "el género, estatus socioeconómico, raza, etnia, habilidad física y mental, orientación sexual, y estatus migratorio, entre otros" (p.279), los/as psicólogos/as no se encuentran exentos de utilizar sesgos o estereotipar, presentar actitudes negativas hacia los pacientes basados en ideas preconcebidas, que se presentan como factores de riesgo en el quehacer profesional en cualquier ámbito de especialización psicológica.

Lo anterior es relevante debido a que, a pesar de propuestas de fortalecimiento de la profesión en universidades chilenas (Calderón et al., 2014), y de que el desarrollo de habilidades de pensamiento como parte de las competencias transversales o genéricas en la formación profesional es un tema que se considera fundamental para los profesionales en ejercicio, no siempre es desarrollado en forma efectiva en los procesos formativos de psicólogos y psicólogas (González, M., González I. \& Vicencio, 2014; Juliá, 2013; Morales, Pino, Ricci, Saavedra \& Zicavo, 2015; Suárez, 2011).

En razón de esto, las instituciones del Consorcio de Universidades del Estado de Chile (CUE) elaboraron un proyecto que busca establecer una estructura común para la formación de profesionales psicólogos/as en base a siete macro-competencias específicas para la disciplina, y diez competencias genéricas, para establecer criterios comunes para el desarrollo de la profesión, acordes a las actuales exigencias de la sociedad (Juliá, 2013).

Dentro de estas competencias específicas, las orientadas al proceso de evaluación psicológica (tanto a nivel individual, grupal e institucional), y algunas competencias genéricas (pensamiento crítico, estratégico, y resolución de problemas), se pueden vincular con la toma de decisiones, y así mismo, a los sesgos, lo que debería ser un tema de preocupación para la formación de futuros profesionales; sin embargo no se conoce en Chile una propuesta de ese tipo. Es por ello, que se planteó como objetivo principal de la presente investigación, conocer los niveles de sesgos de representatividad y confirmación de los futuros profesionales del área de psicología, de manera que se logre determinar una posible caracterización de estos procesos, en estudiantes universitarios de la carrera de Psicología en tres Universidades de la región del Biobío.

\section{Método}

\section{Diseño y tipo de estudio.}

El estudio desarrollado es de corte cuantitativo, ya que se basa en la recolección de datos que se encuentra fundamentada en la medición. Presenta un diseño exploratorio con alcance descriptivo de tipo transeccional (Hernández, Fernández \&. Baptista, 2010).

\section{Participantes.}

La muestra del estudio es de tipo no-probabilística, pues la elección de los elementos no depende de la probabilidad, sino de las características que presenten, las cuales deben estar relacionadas con la investigación (Hernández et al., 2010). Es por esto que se realizó una elección por disponibilidad, pues los participantes se encontraban presentes al momento de aplicar los 
instrumentos.

La población de la presente investigación estuvo compuesta por estudiantes de la carrera de Psicología de tres universidades del sur de Chile: la Universidad del Bío-Bío campus Chillán, Universidad del Desarrollo sede Concepción y Universidad Santo Tomas sede Los Ángeles. A partir de lo anterior, la muestra seleccionada en la investigación fue de 198 alumnos y alumnas de Psicología, que cursaban entre primer y tercer año de esas tres instituciones, de los cuales 133 fueron mujeres (67\%) y 65 hombres (33\%), con una edad que varió entre 17 y 38 años $(M=21$; $\mathrm{DE}=2.8$ ). A nivel de instituciones, la muestra se compuso por 63 estudiantes de la Universidad Santo Tomás (31.8\%), 82 estudiantes de la Universidad del Bío-Bío (41.4\%), y 53 estudiantes de la Universidad del Desarrollo (26.8\%).

\section{Instrumentos.}

El instrumento utilizado en la investigación es llamado Tareas Cognitivas; su estructura se divide en dos grandes tareas, una para medir sesgo de representatividad y otra para sesgo de confirmación. Dicho instrumento ha pasado por un proceso extenso de adaptación, el cual empezó con Kahneman, para luego continuar con Páez et al. (1987); finalmente, se realizó una segunda adaptación por Da Costa (2014), la cual se usó como base de esta investigación. El instrumento cuenta con datos anteriores de confiabilidad de la tarea del sesgo de representatividad, encontrándose en una muestra de estudiantes de pedagogía de la región del Biobio, Chile, un alfa de Cronbach de 0.619 (Ossa, Díaz, Bruna y Cifuentes, 2016).

Para el sesgo de representatividad, se aplicaron dos subtareas. La subtarea uno consistió en seleccionar un valor (entre 0 y 100) sobre la probabilidad de que una persona sea bibliotecario, médico o piloto de avión respectivamente, además se presentó una información que sirvió de distracción, relacionada a aspectos de personalidad. La indicación de la subtarea es:

Elegir la probabilidad entre 0 y 100 de que Esteban sea bibliotecario, médico o piloto de avión. En una muestra de personas con educación superior, la proporción de estas profesiones en esta población es baja, de alrededor del 1\%. La información que se dispone sobre Esteban es la siguiente:

Esteban (nombre del caso) es muy tímido y retraído, siempre servicial, pero poco interesado por la gente o por mundo real; de carácter disciplinado y metódico, necesita ordenarlo todo y tiene obsesión por el detalle.

a. Probabilidad de que sea bibliotecario: (entre 0 y 100).

b. Probabilidad de que sea médico: (entre 0 y 100)

c. Probabilidad de que sea piloto de avión: (entre 0 y 100)

En esta subtarea se espera que se identifique el valor de probabilidad (de cada profesión) señalado explícitamente en el enunciado (1\%) y se desestime la información que genera un estereotipo de personalidad; el valor esperado de estimación en esta subtarea es 0 , valor obtenido de la aplicación de una fórmula matemática, donde el valor elegido por el participante en las profesiones de médico y piloto de avión se suman y dividen por dos, este valor luego es restado al que el participante elige en la probabilidad de bibliotecario, esta debido a que esta profesión es la que arraigaría más el sesgo de la información distractora. Si se obtiene un valor menor a 0 , se está subestimando, y si se obtiene un valor mayor, se sobreestima.

La subtarea dos es similar a la anterior, consiste en determinar la probabilidad entre $0 \mathrm{y}$ 100, que una persona sea ingeniero, presentando una información que sirvió de distracción, relacionada a aspectos de personalidad.

La indicación de la subtarea es: 
Ricardo es un miembro de una muestra donde el $30 \%$ son ingenieros y el $70 \%$ son médicos. Se le ha elegido a él al azar para ser evaluado sobre su rol laboral. La información que se dispone sobre él es la siguiente es la siguiente:

Ricardo es un hombre de treinta años. Está casado y no tiene hijos. Hombre con altas aptitudes, es muy motivado, y una persona muy prometedora en su campo. Sus colegas lo aprecian mucho.

\section{¿Cuál es la probabilidad entre 0 y 100 de que Ricardo sea ingeniero?}

De igual manera que, en la subtarea anterior, se espera que la persona identifique el valor de probabilidad del enunciado (30\%) y desestime la información que genera estereotipo. El valor esperado de estimación en esta subtarea es igualmente 0 , obteniéndose de una fórmula matemática en que, al valor elegido por el particpante, se le resta el valor real del ejercicio; si se obtiene un valor menor a 0 , se está subestimando, y si se obtiene un valor mayor, se sobreestima.

La evaluación del sesgo de confirmación, se hace a través de la tarea de contraste de hipótesis. Esta se presenta a los y las participantes desde la siguiente consigna, la que se encuentra escrita explícitamente en el instrumento aplicado: Imagine que Ud. Debe ayudar a elegir personas para diferentes puestos de trabajo. Para ello es importante usar información para contrastar, y así saber con certeza, sí una persona que es rebelde (para un puesto creativo) y obediente (para un puesto de trabajo rutinario en el que el orden es muy importante) lo es en realidad.

Para responder a la tarea, se otorga una lista de 22 enunciados para la opción A (rebelde), opción B (obediente), opción C (extrovertido/a) y opción D (introvertido/a) respectivamente, donde la persona elige solo 8 que permitan realizar la decisión. La evaluación de esta tarea implica ponderar las situaciones que señalan contrastación del perfil ( 0 punto), y no las que dan confirmación del perfil (1 pto.). Se espera por tanto un puntaje menor a 16, dado que se debiera elegir la información que ayude a contrastar y no a confirmar el perfil, esperando que se seleccionen alternativas equilibradas entre contraste y confirmación, por lo que en cada opción no deberían haber más de cuatro puntos, correspondientes a las confirmaciones, lo que otorga a la prueba total (las cuatro opciones), un ideal de 16 puntos o menos para dar cuenta de que no se cae en sesgo confirmatorio; si se obtienen 17 pts. o más, es señal de que se valora sesgún el sesgo confirmatorio por sobre la contrastación, siendo el valor máximo (cuatro opciones) de 32 pts.

\section{Procedimiento.}

Como mecanismos de consideración ética se aplicó una solicitud de colaboración escrita dirigida a los directores de escuela de las tres instituciones participantes, solicitando posteriormente una entrevista para clarificar dudas y coordinar con los académicos que apoyarían la aplicación del instrumento, que se realizó durante la hora de clases del docente. El instrumento consideró un consentimiento informado escrito en el que se le informaba al participante de los objetivos de la investigación y del instrumento, y se le solicitaba su participación voluntaria y sin presiones. Una vez firmado el consentimiento, se dieron las instrucciones de la aplicación y se tomó el tiempo de ejecución del instrumento que varió entre 15 y 30 minutos aproximadamente.

Para realizar el análisis de datos tanto del nivel de sesgo de representatividad, como del nivel de sesgo de confirmación, se utilizaron estadísticos descriptivos como medidas de tendencia central, medidas de dispersión y de distribución de datos. Siendo necesario una revisión de las características psicométricas del instrumento, se realizó análisis de confiabilidad en la muestra de estudiantes participantes, a través del coeficiente de Cronbach para medir la consistencia interna del instrumento (Gardner, 2003). 
Para el análisis se utilizó estadística descriptiva, mediante estadísticos de tendencia central, dispersión y distribución (media, descviación estándar, asimetría y curtosis). El análisis de la tarea 1 de sesgo de representatividad, tanto de la subtarea 1 como de la subtarea 2 , se realizó mediante distribución en niveles, que fueron definidos mediante cuartiles, en base a los valores obtenidos de la aplicación de la fórmula matemática antes referida. Los niveles fueron Subestimación (valores menores a 0 ), estimación correcta ( 0 a 2), Sobrestimación baja ( 3 a 25$)$, sobrestimación alta (26 a 75) y sobrestimación muy alta (76 y más). Para la tarea 2 de sesgo de confirmación, se realizó el análisis de distribución en porcentajes, atendiendo a si los resultados indicaban valores menores a 16 ( $\sin$ sesgo confirmatorio) o mayores a 17 (sesgo confirmatorio).

Finalmente se analizó la existencia de posibles diferencias entre características demográficas (edad y sexo) mediante el análisis de la prueba t de Student, y de formación (universidad), mediante ANOVA de una vía; en ambos casos se analizó, además, el tamaño de efecto. Para la realización de estos análisis, se utilizó el programa estadístico Paquete Estadístico para las Ciencias Sociales (SPSS).

\section{Resultados}

El análisis de confiabilidad del instrumento Tareas Cognitivas, fue calculada por medio del Alfa de Cronbach en base a los datos tipificados (estandarizados). El resultado para el sesgo de representatividad que fue evaluado en la Tarea 1, es de .609, valor interpretado como una fiabilidad media o regular (Hernández et al., 2010) y bajo el nivel esperado. En cuanto al sesgo de confirmación, evaluado en la Tarea 2, el alfa de Cronbach obtenido es de 0.63, lo que se interpreta así mismo como una fiabilidad media o regular, e igualmente bajo lo esperado.

A nivel global, reuniendo el conjunto de estudiantes de psicología de la muestra, los valores de distribución y dispersión, observados en la tabla 1, muestran valores dentro de parámetros de normalidad, a juzgar por los resultados de asimetría y curtosis, que se encuentran dentro de lo esperado según Bollen y Long (en Núñez-Alonso, Martín-Albo \& Navarro, 2007).

En ambas subtareas del sesgo de representatividad, se observa una media mayor al resultado esperado (valor 0), así como una dispersión de valores mayor en la subtarea uno que en la dos (valores alejándose de cero); mientras que en la tarea 2, del sesgo de confirmación, se observa una media alta (23.9), ya que lo esperado es un valor de 16 o menos) para dar cuenta de que no se está aplicando un sesgo confirmatorio.

Tabla 1.

Valores descriptivos de los instrumentos

\begin{tabular}{llllllll}
\hline & $\mathrm{N}$ & Mínimo & Máximo & Media & D. E & Asimetría & Curtosis \\
\hline Tarea 1.1 & 198 & -83 & 98 & 28.4 & 34.4 & -.305 & .305 \\
Tarea 1.2 & 198 & -46 & 70 & 22.6 & 24.2 & .355 & -.501 \\
Tarea 2 & 198 & 4 & 32 & 23.9 & 5.1 & -.658 & .669 \\
\hline
\end{tabular}

Según los niveles de distribución establecidos más arriba, en el sesgo de representatividad (subtarea 1), solo el 17\% de los alumnos y alumnas de psicología presentan una estimación correcta según los parámetros de la tarea; por otro lado, el $18 \%$ presenta un nivel de sobrestimación baja, 43\% presenta sobrestimación alta, y un 9\% sobrestimación muy alta. Finalmente, se observa que el $10 \%$ de los estudiantes subestima la probabilidad (véase gráfico 1). 


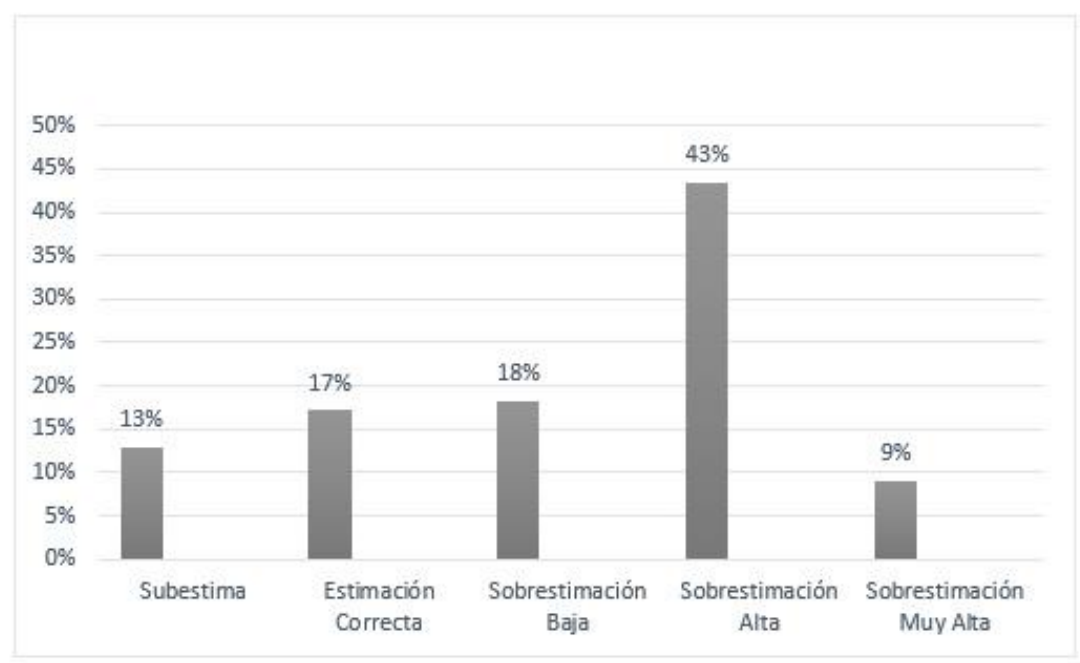

Figura 1. Frecuencia de respuestas según niveles de estimación en sesgo de representatividad (subtarea 1).

En relación con la subtarea dos del sesgo de representatividad, se observa que el $26 \%$ de los estudiantes presenta una estimación correcta según los parámetros de la tarea; por otro lado, el $31 \%$ presenta sobrestimación baja, mientras que el 35\% presenta sobrestimación alta; no existe sobrestimación muy alta (0\%). Finalmente, se observa que el $7 \%$ de los estudiantes subestima (véase gráfico 2).

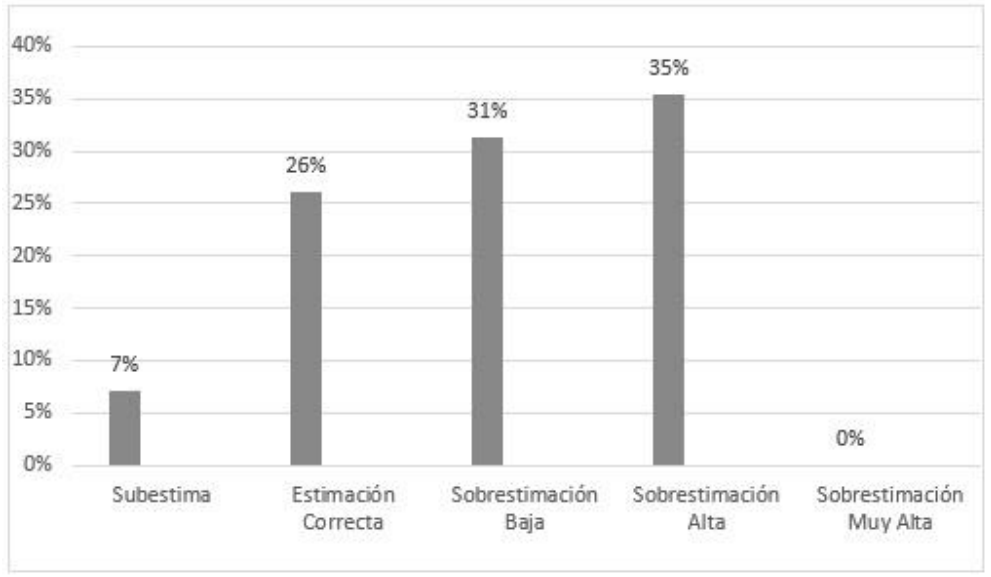

Figura 2. Frecuencia de respuestas según niveles de estimación en sesgo de representatividad (subtarea 2).

En la Tarea 2, de sesgo de confirmación, se puede señalar que solamente un $6 \%$ de la muestra usa el mecanismo cognitivo de contraste para corroborar una idea, seleccionando preguntas que permiten confrontar la información; mientras que el 94\% de los alumnos y las alumnas restante, tienden a buscar información que confirme la situación presentada (Figura 3). 


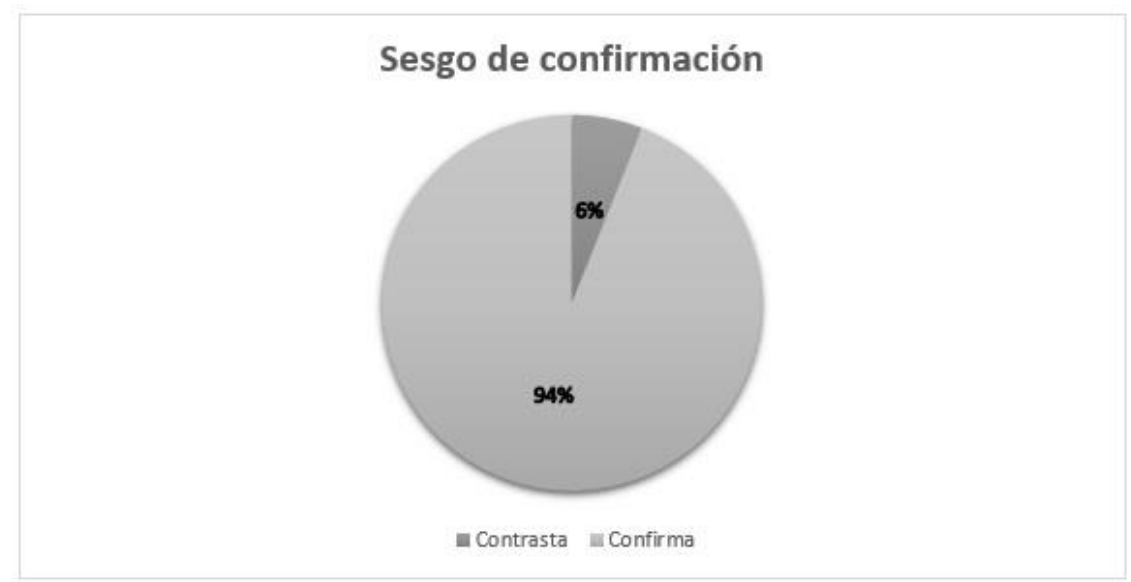

Figura 3. Resultados globales Sesgo de Confirmación.

En relación con la existencia de diferencias en el desempeño de las tareas, en función de edad, sexo y formación, se observa en la tabla 2, que la muestra presenta homocedasticidad en las tres variables, lo que, junto a la presunción de normalidad en la distribución, permitiría sustentar adecuadamente el análisis de diferencias de medias a través de análisis paramétricos.

\section{Tabla 2.}

Valores de homocedasticidad en la muestra según edad, género y formación

\begin{tabular}{|c|c|c|}
\hline Variable/tareas & $\mathrm{F}$ & $p$ \\
\hline \multicolumn{3}{|l|}{ Edad } \\
\hline Tarea 1.1 & .644 & .423 \\
\hline Tarea 1.2 & 1.694 & .195 \\
\hline Tarea 2 & .448 & .504 \\
\hline \multicolumn{3}{|l|}{ Género } \\
\hline Tarea 1.1 & .000 & .992 \\
\hline Tarea 1.2 & .266 & .607 \\
\hline Tarea 2 & .661 & .417 \\
\hline \multicolumn{3}{|l|}{ Formación } \\
\hline Tarea 1.1 & .786 & .457 \\
\hline Tarea 1.2 & 2.190 & .115 \\
\hline Tarea 2 & .486 & .616 \\
\hline
\end{tabular}

El análisis de la prueba $t$ señalado en la tabla 3, muestra que existen diferencias estadísticamente significativas entre el grupo de menor edad (menor a 20 años) y el grupo de 
mayor edad (mayor a 21 años) en la subtarea 1.1 (sesgo de representatividad), donde este último presenta un valor más cercano a 0 , que es lo esperado; sin embargo, no se observaron diferencias significativas en la subtarea 1.2, de representatividad, ni en la de sesgo de confirmación (tarea 2). Por otro lado, no se observan diferencias significativas entre género femenino y masculino, en cuanto al nivel de sesgos, mostrando ambos grupos un nivel similar de desempeño en ambas tareas.

Tabla 3.

Diferencia de medias según edad y género

\begin{tabular}{|c|c|c|c|c|c|c|c|c|}
\hline & Rangos de Edad & & & & & & & \\
\hline & $\begin{array}{l}20 \text { años o menos } \\
\mathrm{n}=106 \\
\text { (media }[\mathrm{DE}] \text { ) }\end{array}$ & $\begin{array}{l}21 \text { años o más. } \\
\mathrm{n}=92 \\
\text { (media }[\mathrm{DE}] \text { ) }\end{array}$ & $\mathrm{t}$ & $\mathrm{gl}$ & $\mathrm{p}$ & $\begin{array}{l}\text { o del } \\
\text { efecto } \\
\text { (d) }\end{array}$ & LI & LS \\
\hline Tarea 1.1 & 33.29 [33.4] & 22.95 [34.9] & 2.12 & 196 & .035 & .30 & .75 & 19.9 \\
\hline Tarea 1.2 & $23.42[23.0]$ & $21.66[25.6]$ & .510 & 196 & $*$ & .07 & -5.05 & 2 \\
\hline Tarea 2 & 23.91 [4.9] & $23.89[5.3]$ & .020 & 196 & & .00 & -1.41 & 8.57 \\
\hline & & & & & .984 & & & 1.44 \\
\hline * Significat & a. .05 & & & & & & & \\
\hline & Sexo & & & & & & & \\
\hline & $\begin{array}{l}\text { Femenino } \\
\mathrm{n}=133 \\
\text { (media }[\mathrm{DE}] \text { ) }\end{array}$ & $\begin{array}{l}\text { Masculino. } \\
\mathrm{n}=65 \\
\text { (media }[\mathrm{DE}] \text { ) }\end{array}$ & $\mathrm{t}$ & $\mathrm{gl}$ & $\mathrm{p}$ & $\begin{array}{l}\text { o del } \\
\text { efecto } \\
\text { (d) }\end{array}$ & LI & LS \\
\hline Tarea 1.1 & $27.18[37.4]$ & 31.15 [33.9] & -.76 & 196 & .44 & .12 & -14.2 & 6.3 \\
\hline Tarea 1.2 & $21.87[29.7]$ & $24.11[23.3]$ & -.60 & 196 & 7 & .08 & -9.4 & 0 \\
\hline Tarea 2 & 23.78 [4.9] & $24.14[5.6]$ & -.46 & 196 & $\begin{array}{l}.54 \\
3\end{array}$ & .07 & -1.87 & $\begin{array}{l}4.9 \\
9\end{array}$ \\
\hline & & & & & .64 & & & 1.2 \\
\hline & & & & & 4 & & & 4 \\
\hline
\end{tabular}

* Significativo a .05

Respecto a la diferencia entre universidades, se observa en la tabla 4 que existen diferencias significativas entre instituciones, en la tarea 1.1 del sesgo de representatividad, y en la tarea 2, correspondiente al sesgo de confirmación. En la subtarea 1 del sesgo de representatividad hay diferencias entre la Universidad del Desarrollo y la Universidad del BíoBío, siendo esta última donde más se presenta el sesgo. Igualmente, en las diferencias de la tarea 2, se observa que la Universidad del Bío-Bío presenta mayor tendencia a la confirmación que las otras dos instituciones. 
Tabla 4.

Diferencias de media entre instituciones

\begin{tabular}{lcccccc}
\hline & N & Media [DE] & F & gl & $p$ & $\begin{array}{c}\text { Tamaño de } \\
\text { efecto }\end{array}$ \\
\hline Tarea 1.1 & & & & & & \\
U. Sto. Tomás & 62 & $27.33[28.77]$ & & & & \\
U. del Bío-Bío & 82 & $37.33[34.0]$ & 6.361 & 2,197 & $.002^{* *}$ & .061 \\
U. del Desarrollo & 54 & $16.49[37.56]$ & & & &
\end{tabular}

Tarea 1.2

$\begin{array}{lllllll}\text { U. Sto. Tomás } & 62 & 20.53[21.04] & & & & \\ \text { U. del Bío-Bío } & 82 & 25.85[25.56] & 1.27 & 2,197 & .283 & .015 \\ \text { U. del Desarrollo } & 54 & 20.06[25.32] & & & & \end{array}$

Tarea 2

$\begin{array}{lllllll}\text { U. Sto. Tomás } & 62 & 22.53[4.75] & & & & \\ \text { U. del Bío-Bío } & 82 & 25.63[4.62] & 8.872 & 2,197 & .000^{* *} & .078 \\ \text { U. del Desarrollo } & 54 & 22.83[5.43] & & & & \end{array}$

** significativo al .01

1 Eta cuadrado parcial

\section{Discusión}

Se corrobora el hecho de que tal como sugieren las investigaciones en el tema (Cortada, 2008; Kahneman et al., 1982; Rodríguez, 2012), existe una alta prevalencia de sesgos cognitivos en los estudiantes, los cuales inciden directamente en que la toma de decisiones se realice de manera automática, siendo muchas veces errónea, ya que no se recoge la información pertinente o adecuada. Esto se evidencia en los resultados encontrados en este estudio, ya que se observa un alto número de estudiantes que presentan niveles de sesgos, tanto de representatividad como de confirmación, dando cuenta que estos estén probablemente naturalizados en los y las estudiantes participantes.

Se observa además que en la tarea 1 (ambas subtareas), existe un alto índice en el nivel de sobrestimación, ya sea de nivel bajo, alto o muy alto, puesto que la mayoría de los estudiantes presentaron valores superiores a cero, que es el valor de estimación correcto; mostrando con ello que los y las estudiantes de psicología, presentarían arraigado el sesgo de representatividad, por lo tanto, a la hora de tomar decisiones, lo harían en base a cuanto representa o se asemeja a sus 
creencias previas, ignorando otra información útil, aun cuando sea explícita (Díaz, Contreras, Batanero \& Roa, 2012; Rodríguez, 2012).

Por otra parte, el sesgo de confirmación, que hace referencia a la tendencia de buscar o seleccionar información que confirme hipótesis previas siendo resistente a información que la contradiga (Muñoz, 2011; Páez et al., 1987), también se presenta muy elevado en los resultados obtenidos en la tarea 2 , con un porcentaje bastante elevado de estudiantes que caerían en estrategias solo confirmatorias y no valorarían las informaciones que permiten contrastar, lo que implicaría que no se esté analizando completamente la situación, sino que existe conformidad en cuanto se observa lo que se desea ver.

Considerando lo descrito con anterioridad, se hace preciso señalar que, al parecer, las/los estudiantes de psicología de las universidades que conformaron la muestra no han desarrollado aún una capacidad de razonar de manera efectiva y no sesgada. Esto puede deberse a múltiples factores, tales como el nivel formativo en que se encuentran, dentro de los primeros tres años de formación, o la orientación de cada casa de estudio en sus bases curriculares, ya que no observa en las mallas curriculares, asignaturas que se ocupen del desarrolle explícito de estas habilidades.

Un aspecto interesante observado en los resultados tiene relación con las escasas diferencias encontradas respecto a la edad, en las que solo se observa que en la subtarea 1.1 de sesgo de representatividad, hay diferencias significativas entre estudiantes mayores a 21 años y los que poseen menor edad, mientras que en la tarea 2.2, y en la de sesgo de confirmación, no se observan diferencias significativas; esto implicaría que los estudiantes con mayor edad logran responder más efectivamente (obviando información tendencial) frente a información menos evidente que los estudiantes de menor edad. Sin embargo, frente a información más explícita, como considerar una línea base de información (el valor de 30\% de ingenieros en la tarea 2.2), el resultado es similar entre ambos grupos de edad;de igual manera ocurriría cuando se solicita analizar un perfil laboral mediante contrastación o confirmación, donde no habrían diferencias respecto a la edad.

Lo anterior podría estar relacionado con dos posibles hipótesis; la primera es que, gracias al proceso de maduración, se tendría menor riesgo de razonar en base a sesgos; la segunda hipótesis sería que, los participantes de mayor edad se encuentran habitualmente en mayores niveles de formación, sea la preparación de las instituciones que va incidiendo en la disminución de esa tarea del sesgo de representatividad.

Si bien no se incorporó en el presente estudio un mecanismo para contrastar dichas hipótesis, por ser solo exploratorio, sería muy interesante continuar con esa línea de indagación para determinar cuál de las dos posibilidades señaladas anteriormente es la que explica mejor estas diferencias.

Asimismo, se hace hincapié en la poca o nula visibilización que existe respecto al proceso de razonamiento, no siendo explícitamente visible el hecho de que se estén potenciando dichas habilidades en las instituciones del estudio; esto podría relacionarse con la implementación de estrategias didácticas tradicionales en las asignaturas de las instituciones, lo que concuerda por lo planteado por Terrén (1996), quien señala que a los alumnos y las alumnas inmersos en una educación caracterizada por la limitación de una enseñanza que coarta capacidades autónomas de decisión, inhibe fuertemente el desarrollo cognitivo.

Lo anterior es más relevante aun al considerar que los sesgos cognitivos incumben en la toma de decisiones (González et al., 2014; Olmo, 2005; Suárez, 2011), lo que gatilla la necesidad de visibilizar la importancia de esta habilidad en el futuro profesional del psicólogo o psicóloga, ya que en él o ella recaen responsabilidades de gran relevancia, sobre todo en la toma de decisiones; por eso es importante tener en cuenta la prevalencia de sesgos cognitivos, debido primordialmente a que trabajan en pro de los demás, con pacientes o usuarios, por eso, se debe 
fortalecer en la formación de los futuros psicólogos o psicólogas el desarrollo del razonamiento en base a información concreta, asimismo, se deben enfocar los aprendizajes que entreguen las instituciones en trabajar y desarrollar el pensamiento crítico en los alumnos y las alumnas en formación.

Dentro de las limitaciones presentadas en la investigación, que corresponden a su diseño exploratorio, la limitación de participantes y la utilización de muestreo por disponibilidad, se considera que la principal se relacionacon los niveles moderados de confiabilidad encontrados en los instrumentos de evaluación de las tareas, lo que secundariamente podría estar relacionado con el número total de participantes de este estudio; por esto se presume que tal vez, con un número mayor de participantes en la muestra, la confiabilidad del instrumento "Tareas Cognitivas" hubiese llegado a ser aceptable o elevada. Por otro lado, el hecho de contar con participantes de una sola región, y el no contar con estudiantes de todos los niveles formativos, también limitaría el alcance de los hallazgos encontrados.

Finalmente, es importante señalar que este estudio podría replicarse para determinar la prevalencia del uso de sesgos en los estudiantes a nivel nacional y analizar cómo las instituciones están abordando esta faceta del desarrollo profesional. Así mismo, podría extenderse indudablemente a otras áreas, tales como comercio, educación, ámbito judicial y área de salud, ya que al igual que las funciones profesionales de los psicólogos y las psicólogas, las áreas anteriormente nombradas, se trabaja también al servicio de las personas, por lo tanto, es importante visibilizar el nivel de sesgos cognitivos en los diferentes profesionales, con el fin de poder ejercer de manera más idónea la profesión.

\section{Referencias}

Cortada, N. (2008). Los sesgos cognitivos en la toma de decisiones. International Journal of Psychological Research, 1(1), 68-73. Doi: https://doi.org/10.21500/20112084.968

Da Costa, S. (2014). Data on representative bias tasks. Unpublished manuscript.

Díaz, C., Contreras, J. M., Batanero, C., \& Roa, R. (2012). Evaluación de sesgos en el razonamiento sobre probabilidad condicional en futuros profesores de educación secundaria. Bolema: Boletim de Educação Matemática, 26(44), 1207-1226. Recuperado de: https://www.ugr.es/ jmcontreras/pages/Investigacion/articulos/2012Bolema.pdf

Díaz-Lázaro, C. (2011). Exploración de prejuicios en los psicólogos: El primer paso hacia la competencia sociocultural. Papeles del Psicólogo, 32(3), 274-281 Recuperado de: https://www.redalyc.org/html/778/77822236008/

González, M., González, I., \& Vicencio, K. (2014). Descripción del rol autopercibido del psicólogo y sus implicancias en los procesos de formación de pregrado. Psicoperspectivas, $\quad 13(1), 108-120 . \quad$ Doi: https://dx.doi.org/10.5027/psicoperspectivas-Vol13-Issue1-fulltext-296

Hernández, R., Fernández, C. \& Baptista, P. (2010). Metodología de la investigación. México: Mc Graw-Hill Interamericana.

Juliá, M. (2013). Competencias de psicólogo en Chile. Propuesta desde las universidades estatales. La Serena: Editorial Universidad de La Serena.

Kahneman, D. Slovic, P., \& Tversky, A. (1982). Judgement under uncertainty: Heuristics and biases. New York: Cambridge University Press.

Martín, L., \& Álvarez, A. (2000). Sesgos cognoscitivos del gerente: su influencia en la toma de decisiones. Revista Cubana de Salud Pública, 26(1), 5-11. Recuperado de: http://scielo.sld.cu/scielo.php?script=sci_arttext\&pid=S0864-34662000000100001

Morris, C., \& Maisto, A. (2001). Psicología. México: Prentice Hall.

Morales, M., Pino, M., Ricci, E., Saavedra, E., \& Zicavo, N. (2015). La formación del Psicólogo en América Latina. En E. Barrero (Coord), Formación en Psicología. Reflexiones y Propuestas desde América Latina (pp. 215-235). Latinoamérica: ALFEPSI.

Muñoz, A. (2011). La influencia de los sesgos cognitivos en las decisiones jurisdiccionales: el factor humano. Una aproximación. InDret, Revista para el análisis de derecho, 2, 1-39. Recuperado de: https://papers.ssrn.com/sol3/papers.cfm?abstract_id=1838370 
Nieto, A. M. (2002). Heurísticos y decisión. En C. Saiz coord., Pensamiento crítico (pp. 215231). Madrid: Ediciones Pirámide.

Núñez-Alonso, J., Martín-Albo, J., \& Navarro, J. (2007). Propiedades psicométricas de la versión española de la escala de motivación deportiva. Revista de Psicología del Deporte, 16(2), 211-223. Recuperado de: https://www.rpd-online.com/article/view/19

Olmo, M. (2005). Prejuicios y estereotipos: un replanteamiento de su uso y utilidad como mecanismos sociales. XXI, Revista de Educación, 13-23. Recuperado de: http://rabida.uhu.es/dspace/bitstream/handle/10272/1957/b15162084.pdf?sequence=1

Ossa, C., Díaz, A., Bruna, D., \& Cifuentes, F. (2016). Relación entre habilidades de indagación, razonamiento probabilístico y sesgo de representatividad en estudiantes de pedagogía. Innovare. Revista Electrónica de Educación Superior, 1(2), 109-128. Recuperado de: http://innovare.udec.cl/wp-content/uploads/2017/01/0719-7500.2016.11.pdf

Páez, D., Villarreal, M., Echeverría, A., \& Valencia, J. (1987). Cognición social: Esquema y función cognitiva aplicada al mundo social. En D. Páez. (Eds.), Pensamiento, individuo y sociedad. Cognición y representación social. Madrid: Editorial Fundamentos.

Rivas, M. (2008). Procesos cognitivos y aprendizaje significativo. Documento de trabajo $\mathrm{N}^{\circ} 19$. Madrid: Consejería de Educación de la Comunidad de Madrid. Recuperado de: http://www.madrid.org/bvirtual/BVCM001796.pdf

Rodríguez, E. (2012). Toma de decisiones: la economía conductual (Tesis de maestría). Universidad de Oviedo, España. Recuperado de: http://digibuo.uniovi.es/dspace/bitstream/10651/13074/1/Trabajo\%20fin\%20de\%20m \%C3\%A1ster\%20Eduardo\%20Rodr\%C3\%ADguez\%20Quintana.pdf

Suárez, X. (2011). Valoración de las Competencias de Psicología: Estudio exploratorio en muestras de Estudiantes y Profesionales Activos. Revista de Psicología, 20(1), 73 - 102. Doi: https://dx.doi.org/10.5354/0719-0581.2011.13727

Terrén, E. (1996). Las aulas desencantadas. Max Weber y la educación. Política y Sociedad, 21, 133-148. Recuperado de: http://revistas.ucm.es/index.php/POSO/article/viewFile/POSO9696130133A/25456

Tversky, A., \& Kahneman, D. (1983). Extensional versus Intuitive Reasoning: The conjunction fallacy in probability judgement. Psychological Review, 90(4), 293-315. Recuperado de:

http://faculty.econ.ucdavis.edu/faculty/nehring/teaching/econ106/readings/Extensional $\% 20$ Versus\%20Intuitive.pdf 